\title{
How to derive the analytical capacity model for not-conventional urban roundabouts
}

\author{
O. Giuffrè ${ }^{1}$, A. Granà ${ }^{1}$, T. Giuffrè ${ }^{2}$ \& R. Marino ${ }^{1}$ \\ ${ }^{I}$ Department of Road Infrastructure Engineering, \\ Palermo University, Italy \\ ${ }^{2}$ Department of Aeronautics Technologies and Infrastructures, \\ Palermo University, Italy
}

\begin{abstract}
The inapplicability of current methods for analyzing the operations of notconventional urban roundabouts (i.e. not referable to standard schemes) seriously hampers the assessment of their operational performances. Moreover, local constraints and the road network structure have produced lots of geometric layouts that make it hard to propose a framework to explain the performances of not-conventional roundabouts. For these intersections it is also hoped to have scientifically based methods to analyze operations with proper reliability. Starting from these considerations, this paper shows the conceptual path followed to analyze the operations of not-conventional roundabouts along an arterial of Palermo City. The examined intersections have suggested a theoreticexperimental approach that balances the need both to match field observations and to have a general criterion to determine performances. A case study application allows one to explain how to derive the analytical capacity model from field data. Moreover the comparison of results to those derived by models for conventional schemes informs us about the effect on capacity caused by a more realistic operational pattern such as that observed at multilane-largediameter roundabouts.
\end{abstract}

Keywords: operations, headway, multilane large diameter roundabouts.

\section{Introduction}

The subject of the present research concerns the analysis of traffic operations at particular urban circular intersections resulting in not-conventional roundabouts 
(i.e. they are not referable to typical schemes by geometric and functional characteristics), for which ordinary capacity models fall down and are not applicable. In particular the case of multilane-large-diameter roundabouts (i.e. characterized by a central island with a large diameter and by two or more lanes at entry and a circulatory roadway) has been explored. Field observations at a sample of multilane-large-diameter roundabouts (MLLD roundabouts in the following) have shown that users (both entering from approaches and streaming on the circulating carriageway) as often as not transgress the nominal regulation of the right-of-way, but they negotiate it with conflicting vehicles. In this pattern, as well as operations occurring in all-way-stop-controlled intersections [1], the right-of-way happens to vehicles entering from the approach and vehicles streaming in the circulating lanes in turn.

The main objective of this paper is to develop from field observations an operational analysis method to evaluate performances at MLLD roundabouts. The application to a case study can allow us to illustrate how to derive the analytical capacity model through an exploratory analysis of experimental data. The description of the procedure implemented to obtain capacity computations at MLLD roundabouts will be preceded by a brief analysis of the macroscopic observations of traffic conditions at the examined type of roundabouts, in order to deduce the main analogies with operations at all-way-stop-controlled intersections [1].

\section{Operational conditions at multilane-large-diameter roundabouts}

Traffic conditions at not-conventional MLLD roundabouts selected as case studies have been videotaped to examine the elements of operations that can influence performances. It has been observed that behavioral aspects are considerable as regards frequent inversions of right-of-way between entering and circulating vehicles even though the entry manoeuvre is regulated by the giveway sign. In particular, field observations have highlighted:

- circulating vehicles along the circulatory roadway give priority (or they are forced to give priority) to entering vehicles and they stop before proceeding into the intersection at an ideal (not-existing) stop line;

- circulating vehicles tend to give priority even if entering vehicle flows are low and they arrange themselves in parallel rows before moving again;

- entering vehicles are able to force circulating vehicles to wait, even if the last ones are characterized by high volumes.

In every case, vehicles from the entry approach give the priority to circulating vehicles in an advanced position regarding the stop line materialized on the road, waiting behind an ideal stop line on the circulatory roadway.

These observations allowed to deduce that operational conditions at MLLD intersections are similar to that ones occurring at all-way-stop-controlled intersections (where drivers on all approaches have to stop before proceeding into the intersection) and follow a pattern of consensus of right-of-way that alternates among entry and circulating drivers. The main consequence is that 
traffic operations at the examined not-conventional roundabouts cannot be explained through models based on gap-acceptance theory; on the contrary, an operational analysis method can be developed starting from the methodology for all-way-stop-controlled intersections, as it will be described in the following paragraph.

\section{The analytical capacity model for urban not-conventional multilane large diameter roundabouts}

In order to derive the analytical capacity model for urban MLLD roundabouts, as those examined in this paper, a theoretic-experimental approach to analyze traffic operations and drivers' behavioural parameters has been developed. Similarly to the method proposed by HCM 2000 for all-way-stop-controlled intersections [1], a calculation algorithm to evaluate performances at each MLLD roundabout approach has been implemented following subsequent computational steps. Each approach is analyzed as an all-way-stop-controlled intersection approach, where each conflicting approach is a one-way street.

The description of the operational analysis method developed for capacity computations at a three circulating/entering lanes MLLD roundabout will be preceded by an overview of the methodological base of the procedure.

\subsection{Overview of the methodology}

The affinities between operational conditions at MLLD roundabouts and at allway-stop-controlled intersections suggested modelling the operations at MLLD roundabouts starting from a discrete number of saturation headway values. Each saturation headway reflects a different degree of conflict between the subject approach driver (i.e. by the driver at the stop line of the entry approach or at the similar ideal stop line sited on the circulatory roadway) and vehicles on the conflicting approach. Several studies [1-3] show that the departure headway between consecutively departing subject approach vehicles is the expected value of the saturation headway distribution:

$$
h_{d}=\sum_{i=1}^{n} P(i) \times h_{s i}
$$

where:

$\mathrm{P}(\mathrm{i})=$ the probability of the degree-of-conflict case $i$;

$\mathrm{h}_{\mathrm{si}}=$ saturation headway for the degree-of-conflict case $i$, given the traffic stream and geometric conditions of the intersection approach;

$i=$ each combination of the $\mathrm{n}$ degree-of-conflict cases.

Apart from the consequences in the practical applications, the difficulty of this methodological approach is evident considering that basic behavioural parameters (i.e. the saturation headways) necessary to develop a capacity model are difficult to be observed, because they often elude direct observations. A procedure to draw saturation headways by lane from observations of traffic conditions at not-conventional roundabouts has been proposed by the authors in 
a previous work [4]. In particular, the presence of response correlation has required the unknown parameters to be estimated through a marginal model, based on the degree of conflict experienced by the subject approach driver; this model has been developed by the well-known Generalized Estimating Equations models [5] and then calibrated from macroscopic observations of traffic conditions. Differently from AWSC intersections, at a generic approach of a MLLD roundabout only two degree-of-conflict cases can be faced by entering (or circulating) vehicles. The first one (degree-of-conflict case 1/0) chances when no vehicle is present at circulating lanes (or at entry); the second one (degree-of-conflict case $3 / *$ ) chances when one or more vehicles at circulating lanes (or at entry) are present and it can be expanded to three under-cases depending on the number of vehicles being on the conflicting approach.

The probability of occurrence for each degree-of-conflict case $P(i)$ can be computed using the following equation:

$$
P(i)=\prod_{L z} P\left(a_{L z}\right)
$$

where:

$L z=$ each lane of the conflicting approach; $z=1,2,3$;

$a_{L z}=1$ indicates a vehicle present; 0 indicates no vehicle present by lane;

$P\left(a_{L z}\right)=$ probability of $\mathrm{a}_{\mathrm{Lz}}$

Values of $\mathrm{a}_{\mathrm{Lz}}$ for each lane in each combination $i$ are listed in Table 1; the probability there is or not a vehicle at a generic lane of the conflicting approach is also defined in Table 1 in which $V_{L z}$ is the lane flow rate at lane $\mathrm{L}_{\mathrm{z}}$.

Table 1: $\quad$ Probability of $\mathrm{a}_{\mathrm{Lz}}$.

\begin{tabular}{|c|c|l|}
\hline$a_{L z}$ & $V_{L z}$ & $P\left(a_{L z}\right)$ \\
\hline 1 & 0 & 0 \\
\hline 0 & 0 & 1 \\
\hline 1 & $>0$ & $x_{L z}$ \\
\hline 0 & $>0$ & $\left(1-x_{L z}\right)$ \\
\hline
\end{tabular}



According to HCM 2000, at the generic subject approach, the probability of the degree-of-conflict case $i$ shown in Table 1 is computed starting from the degree of utilization $(x)$ of each lane in the conflicting approach; when arrival rate in the considered lane is $V_{L z}(\mathrm{veh} / \mathrm{s})$, the degree of utilization is:

$$
x=\frac{V_{L z} \times h_{d, L z}}{3600}
$$

Table 2 shows the possible combinations of degree-of-conflict cases for a three circulating/entering lanes MLLD approach and their probability of occurrence. 
In total, for a three circulating/entering lanes MLLD approach just two degree-of-conflict cases, corresponding to eight combinations, have to be considered.

Table 2: $\quad$ Degree-of-conflict cases and probability of occurrence.

\begin{tabular}{|c|c|c|c|c|c|c|}
\hline \multirow{3}{*}{\multicolumn{2}{|c|}{ degree-of-conflict case }} & \multicolumn{4}{|c|}{ approach } & \multirow{3}{*}{ probability of occurrence } \\
\hline & & \multirow{2}{*}{$\frac{\text { subject }}{\mathrm{L}_{\mathrm{z}}}$} & \multicolumn{3}{|c|}{ conflicting } & \\
\hline & & & $\mathrm{L}_{1}$ & $\mathrm{~L}_{2}$ & $\mathrm{~L}_{3}$ & \\
\hline 1 & $1 / 0$ & 1 & 0 & 0 & 0 & $\mathrm{P}\left(\mathrm{C}_{1 / 0}\right)=\left(1-\mathrm{x}_{\mathrm{L} 1}\right)\left(1-\mathrm{x}_{\mathrm{L} 2}\right)\left(1-\mathrm{x}_{\mathrm{L} 3}\right)$ \\
\hline 2 & $3 / 1 \mathrm{a}$ & 1 & 1 & 0 & 0 & $\mathrm{P}\left(\mathrm{C}_{3 / 1}\right)_{\mathrm{a}}=\mathrm{x}_{\mathrm{L} 1}\left(1-\mathrm{x}_{\mathrm{L} 2}\right)\left(1-\mathrm{x}_{\mathrm{L} 3}\right)$ \\
\hline 3 & $3 / 1 \mathrm{~b}$ & 1 & 0 & 1 & 0 & $\mathrm{P}\left(\mathrm{C}_{3 / 1}\right)_{\mathrm{b}}=\left(1-\mathrm{x}_{\mathrm{L} 1}\right) \mathrm{x}_{\mathrm{L} 2}\left(1-\mathrm{x}_{\mathrm{L} 3}\right)$ \\
\hline 4 & $3 / 1 \mathrm{c}$ & 1 & 0 & 0 & 1 & $\mathrm{P}\left(\mathrm{C}_{3 / 1}\right)_{\mathrm{c}}=\left(1-\mathrm{x}_{\mathrm{L} 1}\right)\left(1-\mathrm{x}_{\mathrm{L} 2}\right) \mathrm{x}_{\mathrm{L} 3}$ \\
\hline 5 & $3 / 2 \mathrm{a}$ & 1 & 1 & 1 & 0 & $\mathrm{P}\left(\mathrm{C}_{3 / 2}\right)_{\mathrm{a}}=\mathrm{x}_{\mathrm{L} 1} \mathrm{x}_{\mathrm{L} 2}\left(1-\mathrm{x}_{\mathrm{L} 3}\right)$ \\
\hline 6 & $3 / 2 b$ & 1 & 1 & 0 & 1 & $\mathrm{P}\left(\mathrm{C}_{3 / 2}\right)_{\mathrm{b}}=\mathrm{x}_{\mathrm{L} 1}\left(1-\mathrm{x}_{\mathrm{L} 2}\right) \mathrm{x}_{\mathrm{L} 3}$ \\
\hline 7 & $3 / 2 \mathrm{c}$ & 1 & 0 & 1 & 1 & $\mathrm{P}\left(\mathrm{C}_{3 / 2}\right)_{\mathrm{c}}=\left(1-\mathrm{x}_{\mathrm{L} 1}\right) \mathrm{x}_{\mathrm{L} 2} \mathrm{x}_{\mathrm{L} 3}$ \\
\hline 8 & $3 / 3$ & 1 & 1 & 1 & 1 & $\mathrm{P}\left(\mathrm{C}_{3 / 3}\right)=\mathrm{x}_{\mathrm{L} 1} \mathrm{x}_{\mathrm{L} 2} \mathrm{x}_{\mathrm{L} 3}$ \\
\hline
\end{tabular}

Note: 1 denotes presence of a vehicle; 0 denotes absence of vehicle.

For each lane in the subject approach, headways between consecutively departing subject approach vehicles can be expressed starting from equation 1 as follows:

$$
\begin{aligned}
\left(h_{d}\right)_{L z}= & \sum_{i=1}^{8} P(i) \cdot\left(h_{s i}\right)_{L z}=P\left(C_{1 / 0}\right) \cdot\left(h_{S 1 / 0}\right)_{L z}+\sum_{i=2}^{4} P\left(C_{3 / 1}\right)_{i} \cdot\left(h_{S 3 / 1}\right)_{L z, i}+ \\
& +\sum_{i=5}^{7} P\left(C_{3 / 2}\right)_{i} \cdot\left(h_{S 3 / 2}\right)_{L z, i}+P\left(C_{3 / 3}\right) \cdot\left(h_{S 3 / 3}\right)_{L z}
\end{aligned}
$$

\subsection{Capacity computational steps}

Starting from the above outlined methodological base, a calculation algorithm has been implemented to evaluate the performances at each MLLD roundabout approach. First of all, for an assigned set of traffic and geometric conditions, the algorithm allows one to estimate departure headways $\left(h_{d}\right)_{L z}$, i.e. the headways between consecutively departing subject approach vehicles by lane; the iteration of the procedure by increasing flow rate allows one to estimate capacity at each entry/circulating lane. The entire procedure consists of the following subsequent steps:

1. data input: the data for evaluating $h_{d, L z}$ are: i) the number of entering and circulating lanes; ii) the traffic volumes, in total and by lane; iii) the turning movements of interest, when shared lanes are present. The specification of traffic data by vehicle direction is also useful to define the saturation headway values $\left(h_{s i}\right)$; 
2. probability states: the step regards: i) the specification of the degree-ofconflict cases; ii) the estimate of the degree of utilization by lane; iii) the calculation of the probability of each degree-of-conflict case;

3. saturations headways: as sum of base saturation headway and saturation headway adjustment factor;

4. the computation of $\left(\mathrm{h}_{\mathrm{d}}\right)_{\mathrm{Lz}}$ by each lane of the subject approach (i.e. the entry or the circulating lane in turn). Considering the interdependence of the traffic flows on the approaches, this computation creates the need for iterative calculations. So, the departure headway computed in each iteration for an approach, considered as the subject approach, is used as initial departure headway in the next iteration for the conflicting approach (when this becomes the subject approach in turn). The process is repeated until the change in headways between two successive iterations is less than $0.1 \mathrm{~s}$;

5. the approach capacity computation: according to Kyte et al. [3] and to indications about this subject reported in HCM 2000 [1], the capacity of a lane at MLLD roundabouts can be defined as the maximum throughput on an approach given the distribution of flow rates by lane on the other intersection approach. Therefore, the capacity of the approach is computed under the assumption that the flows on the conflicting approach lane are constant. The given flow rate on the subject lane is increased under an assigned hypothesis of increment, maintaining constant the traffic volume at the conflicting approach and in the adjacent lanes, too; when the degree of utilization on any one approach lane reaches 1 , the final value of the subject approach flow rate is the maximum throughput or capacity of the considered lane.

\subsection{The application of the capacity model to a case study}

To illustrate the iterative procedure for capacity calculations as above outlined, a three circulating/entering MLLD roundabout selected as case study from Palermo City road network has been examined. The exploratory field data analysis at this intersection (at the entries and at the circulatory roadway), as well as the estimates of model parameters and model validation, are reported in a previous work [4]. The saturation headways $\left(\mathrm{h}_{\mathrm{si}}\right)$, by lane, are determined through the procedure developed to draw them from traffic observations and they are computed as the sum of a base saturation headway, by lane, and a saturation headway adjustment factor. This factor is related to through movements (computed as the share of these manoeuvres in the considered lane) since it showed to have some influence on the departure headway. So, saturation headways are computed by the following:

$$
h_{s i, L z}=h_{\text {si-base }, L z}+h_{T H_{i, L z}} P_{T H_{i, L z}}
$$

where:

$h_{\text {si-base, } L z}=$ saturation headway for the degree-of-conflict case $i$, by lane;

$h_{T H, L z}=$ headway adjustment for through traffic movements, by lane;

$P_{T H, L z}=$ proportion of through traffic movements on the approach, by lane. 
The saturation headways at the roundabout selected as case study are reported in Table 3. Based on field observations, the estimates in Table 3 have been obtained considering equation (4) as a regression model and assuming that saturation headways are observable only in the degree-of-conflict cases (1/0) and

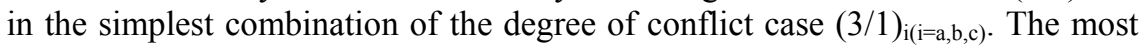
appropriate regressors of the residual response variate (i.e. unobservable parameters $h_{\mathrm{si}}$ ) have been estimated considering together the probability of occurrence of degree-of-conflict cases $(3 / 2)_{\mathrm{i}(\mathrm{i}=\mathrm{a}, \mathrm{b}, \mathrm{c})}$ and $(3 / 3)$. The predictive capacity of the regression model has been validated starting from traffic observations at approach lanes.

Table 3: $\quad$ Saturation headways by degree-of-conflict case, approach and lane.

\begin{tabular}{|c|c|c|c|c|c|c|c|}
\hline \multirow{2}{*}{ DOC case } & \multirow{2}{*}{$\mathrm{h}_{\mathrm{s}}$} & \multicolumn{3}{|c|}{ circulatory roadway } & \multicolumn{3}{c|}{ entry } \\
\cline { 3 - 8 } & & $\mathrm{L}_{1}$ & $\mathrm{~L}_{2}$ & $\mathrm{~L}_{3}$ & $\mathrm{~L}_{1}$ & $\mathrm{~L}_{2}$ & $\mathrm{~L}_{3}$ \\
\hline $\begin{array}{c}1 / 0 \text { or } \\
(3 / 1)_{\mathrm{i}}\end{array}$ & $\mathrm{h}_{\text {si-base }}$ & 1.99 & 1.42 & 1.13 & 1.73 & 1.16 & 0.87 \\
\cline { 2 - 8 } & $\mathrm{h}_{\mathrm{TH}}$ & - & - & - & - & - & - \\
\hline $\begin{array}{c}(3 / 2)_{\mathrm{i}} \text { plus } \\
(3 / 3)\end{array}$ & $\mathrm{h}_{\text {si-base }}$ & 8.53 & 5.77 & 4.10 & 6.53 & 3.77 & 2.10 \\
\cline { 2 - 8 } & $\mathrm{h}_{\mathrm{TH}}$ & 1.73 & 1.73 & 1.73 & 1.73 & 1.73 & 1.73 \\
\hline
\end{tabular}

To illustrate the way of operating and working limits at the not-conventional roundabout selected as case study, a representation of capacity versus conflicting volumes can be obtained turning to particular hypotheses: i) three lanes at entry and three lanes at circulating lanes; ii) the outer lane at entry approach is not conditioned by any conflicting volumes; iii) the initial traffic volumes both at entry and at circulatory carriageway have the same total values and they are equally shared among lanes. Table 4 shows some results of the proposed computational algorithm in the above specified set of conditions. The values in the table refer only to lanes $\mathrm{L}_{1}$ and $\mathrm{L}_{2}$; the outer entry lane $\left(\mathrm{L}_{3}\right)$ has been disregarded because vehicular movements are thought to follow a different pattern of right-of-way (see above hypothesis ii).

We remark that results presented in Table 4 have to be read considering the particular set of conditions in which they have been obtained. For example, when the traffic volume at the entry lane $L_{1}$ reaches a capacity value equal to 1870 $\mathrm{veh} / \mathrm{h}$ the corresponding total circulating volume is equal to $500 \mathrm{veh} / \mathrm{h}$ and it is shared equally among the three lanes; moreover, while the volume on the examined subject approach lane (lane $\mathrm{L}_{1}$ ) increases incrementally, the flows on the other approach lane is constant and equal to the initial volume values $(250$ $\mathrm{veh} / \mathrm{h}$ ). Figure 1 shows the comparison among the capacity values at entry approach for the examined three entering/circulating lane MLLD roundabout and:

- capacity values as computed by Kimber linear model [6] at very large diameter roundabouts;

- approach capacity modelled by Brilon and $\mathrm{Wu}$ [7], as referred by the HBS2001 [8]. 
Table 4: $\quad$ Entry lane capacity vs. conflicting volume at a MLLD roundabout.

\begin{tabular}{|c|c|c|}
\hline Qcr $[\mathrm{veh} / \mathrm{h}]$ & Entry lane $\mathrm{L}_{1}[\mathrm{veh} / \mathrm{h}]$ & Entry lane $\mathrm{L}_{2}[\mathrm{veh} / \mathrm{h}]$ \\
\hline 100 & 2090 & 3090 \\
\hline 200 & 2060 & 3060 \\
\hline 300 & 2010 & 2990 \\
\hline 400 & 1960 & 2860 \\
\hline 500 & 1870 & 2710 \\
\hline 600 & 1780 & 2540 \\
\hline 700 & 1670 & 2290 \\
\hline 800 & 1520 & 1940 \\
\hline 900 & 1330 & 1470 \\
\hline 1000 & 1100 & 1120 \\
\hline 1100 & 860 & 860 \\
\hline 1200 & 670 & 670 \\
\hline Data fit: & $\mathrm{y}=-0.0012 \mathrm{x}^{2}+0.2314 \mathrm{x}+2063.2$ & $\mathrm{y}=-0.0017 \mathrm{x}^{2}-0.186 \mathrm{x}+3182.3$ \\
$\mathbf{R}^{2}=\mathbf{0 . 9 9 8 4}$ & $\mathbf{\mathbf { R } ^ { 2 }}=\mathbf{9 8 8 1}$ \\
\hline
\end{tabular}

Note: “cr" in $Q_{c r}$ means circulatory carriageway

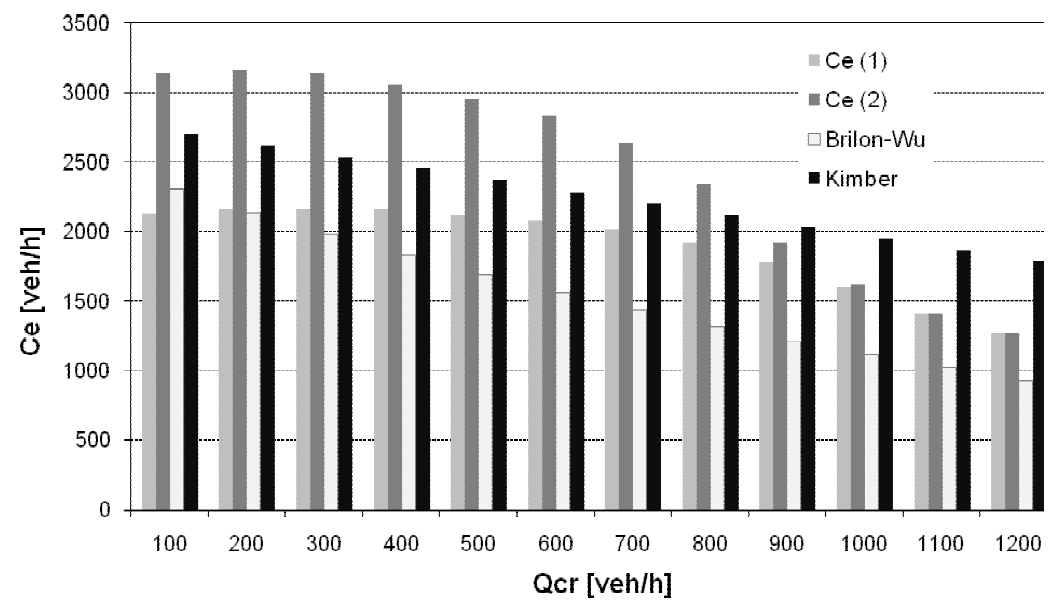

Figure 1: Comparing model capacity to experimental data at a multilane roundabout. Note: capacities computed by the Brilon-Wu (1996) model are related to $\mathrm{Ne} / \mathrm{Ncr}=2 / 3$.

Models used for comparison turn to the total circulating flow rate to determine the total entry capacity per approach. The UK Linear Regression model estimates are higher than the capacity model as formulated by Brilon-Wu in the whole range of variation of the circulating flow rate. As shown, when the total circulating volume $\left(\mathrm{Q}_{\mathrm{cr}}\right)$ is equal to $500 \mathrm{veh} / \mathrm{h}$ the corresponding 
experimental approach capacities $C_{\mathrm{e}}(1)$ and $\mathrm{C}_{\mathrm{e}}(2)$ are equal to $2120 \mathrm{veh} / \mathrm{h}$ and $2960 \mathrm{veh} / \mathrm{h}$, respectively. In particular, the value $2120 \mathrm{veh} / \mathrm{h}$ has been obtained adding the capacity value reached by the lane $\mathrm{L}_{1}$ during the incremental phase and equal to $1870 \mathrm{veh} / \mathrm{h}$ to the initial traffic volume $(250 \mathrm{veh} / \mathrm{h})$, maintained constant in the adjacent entering lane $\mathrm{L}_{2}$. Analogous considerations regard experimental values $\mathrm{C}_{\mathrm{e}}(2)$.

As it is shown, high capacity values correspond to low conflicting volume and, when the conflicting volumes increase experimental capacity values tend to decrease faster than it can be observed at a conventional roundabout. Basing on what shown by the graph, the gain of capacity imputable to the consensus of right-of-way between entering and circulating vehicles at the examined MLLD roundabout interests model values from low-to-high circulating flows. Comparing capacity models for conventional roundabouts to data obtained by model capacity for MLLD roundabouts allows us to deduce that proposed method seems to be able for qualifying and quantifying capacity parameters at particular not-conventional roundabouts.

\section{Conclusions}

Modelling traffic operations at not-conventional roundabouts can be extremely complex both in relation to vehicles disregarding the priority rule and to free movements of vehicles not conditioned by conflicting streams. The analogy established between multilane-large-diameter roundabouts and all-way-stopcontrolled intersections let us to develop an operational analysis method for interpreting (both in qualitative terms and in quantitative ones) experimental data about intersection operations and to propose a calculation algorithm for evaluating the performances at a MLLD roundabout approach by subsequent computational steps. The application to the not-conventional roundabout examined as case study allowed us to illustrate how to derive model specification through exploratory analysis of field observations. It has to be highlighted that the proposed method can easily be adapted to specific intersection layouts and to account for factors able to affect operations (the lane occupied by vehicles at approaches, type of vehicular movements, presence of heavy vehicles, etc.). Accessing to these parameters is the necessary stage for developing an analytical model able to estimate delays experienced by the users at not-conventional roundabouts.

\section{References}

[1] HCM 2000, TRB Highway Capacity Manual. Transportation Research Board, National Research Council, Washington, D.C., 2000.

[2] Richardson, A., A delay Model for Multiway Stop-Sign Intersections. Transportation Research Record 1112, TRB, Washington, D.C., pp. 107114, 1987.

[3] Kyte, M., Tian, Z., Mir, Z., Hameedmansoor, Z., Kittelson, W., Vandehey, M., Robinson, B., Brilon, W., Bondzio, L., Wu, N., \& R. Troutbeck. 
NCHRP Web Doc 6: Capacity and level of service at unsignalized intersections, Final Report, Vol. 2, All-Way Stop-Controlled Intersections, http://www.nap.edu/books/nch006/html. The National Academies of Sciences, 1997.

[4] Giuffrè, O., Granà, A., Giuffrè, T. \& Marino, R., Modeling traffic operations and driver' behavioral parameters at not-conventional roundabouts. A theoretic-experimental approach. Proc. of the 86th TRB Annual Meeting. Washington, D.C., U.S.A, pp. 1-16, 2007.

[5] Hardin, J. W. \& Hilbe, J. M., Generalized estimating equations. Chapman and Hall/CRC Press. Boca Raton, FL, 2003.

[6] Kimber, R.M., The traffic capacity of roundabouts, TRRL Laboratory, Report 942, Crowthorne, Berkshire, UK, 1980.

[7] Brilon, W. \& Wu, N., User Manual of KREISEL, Germany, 1996.

[8] FGSV 2001. Handbook for assessing road traffic facilities. Koln: Forschungsgesellschaft fur das Strassen und Verkehrswesen HBS 2001. 